\title{
LIMITATIONS OF THE ISOLATED GP-STN NETWORK
}

\author{
Ian M. Stanford, Krim C. Loucif, Claire L. Wilson, Diana Cash and \\ Michael G. Lacey*
}

\section{INTRODUCTION}

Activity of the globus pallidus (GP) - subthalamic nucleus (STN) network plays a pivotal role in movement disorders of the basal ganglia. Thus, during dopamine depletion, in idiopathic and animal models of Parkinson's disease, there is an increase in oscillatory burst firing and synchronisation of GP and STN neurons (Bergman et al 1994, Nini et al 1995 Raz et al 2000, 2001). This activity is transmitted to basal ganglia output stations contributing to the excessive inhibition of the thalamocortical motor loop and thus, the symptoms of akinesia and rigidity (Albin et al. 1989, DeLong 1990) and muscle tremor (Filion and Tremblay 1991, Bergman et al 1994, Magnin et al 2002). But how does this activity arise and can it be sustained in the isolated GP-STN network?

Using a culture preparation, Plenz and Kitai (1999) proposed that the GP and STN form a central pacemaker responsible for oscillatory activity in the basal ganglia. Brief applications of GABA (to mimic pallidal synaptic input) were able to promote burst firing in the STN, through the de-inactivation of a low-threshold calcium conductance, while STN activity reverted to tonic firing once the pallidal input was disconnected. Therefore, it appeared that viable reciprocal connectivity between the GABAergic GP and glutamatergic STN may be sufficient for the generation and recruitment of the STN rebound burst activity and thus support regenerative oscillatory activity (Plenz and Kitai 1999; Bevan et al. 2000).

However, in vivo experiments have indicated that rhythmic activity in GP and STN neurons is driven by the cortex (Magill et al. 2000), with dopamine depletion sensitizing the system further (Magill et al. 2001). Therefore, aside from in the culture preparation, is there evidence that the GP and STN in isolation can maintain such activity? Indeed, such a hypothesis would gain credibility if reproduced in situ. This chapter details the search for such evidence in a mouse slice preparation in which reciprocal connectivity between the GP and the STN is maintained.

*Ian M. Stanford, Krim C. Loucif, Claire L. Wilson. School of Life and Health Sciences, Aston University, Birmingham B4 7ET. U.K. Diana Cash, Neuroimaging Research Group, Institute of Psychiatry, London SE5 8AF, U.K. Michael G. Lacey, The Division of Neuroscience, The Medical School, The University of Birmingham, Edgbaston, Birmingham. B15 2TT. U.K. 


\section{I.M.STANFORD ET AL.}

\section{THE PREPARATION}

In order to optimize the extent of the interconnectivity between the GP and the STN we developed a parasagittal mouse slice preparation cut $20^{\circ}$ to the midline in which the distance between the two brain nuclei is about $1 \mathrm{~mm}$. Connectivity was then demonstrated morphologically, using biocytin-tracing techniques, and by electrical stimulation in either nucleus to evoke inhibitory GABA or excitatory glutamate mediated post-synaptic currents (I/EPSCs). In addition, spontaneous IPSCs (which can only emanate from the GP) and EPSCs (either from the cortex, pedunculopontine nucleus or thalamus) were also observed. This preparation was then used to directly investigate the role of GABA and glutamate release in shaping and modulating neuronal activity in control and MPTP-treated mice.

\subsection{Biocytin Tracer Studies}

$300 \mu \mathrm{m}$ thick slices were obtained from CB57BL/6JGL male mice 21-40 days of age. In order to assess the anatomical connectivity between the GP and STN we exploited the retrograde and anterograde transport of the neuroanatomical tracer biocytin. Biocytin (Sigma Chemicals, Poole, UK) was mixed with 20\% gelatin (Fisher Scientific, Loughborough, UK) in Tris-buffered saline to a final concentration of 50\%. Pellets were injected into GP and STN in horizontal (cut $10^{\circ}$ to true) and parasagittal (cut $20^{\circ}$ to the midline) sections using either a $1 \mu$ Hamilton syringe or glass pipette attached to a Picospritzer II (General Valve Corporation, NJ, USA) pressure ejection system. Following 8-10 hours of continuous perfusion with aCSF, slices were fixed in $4 \%$ paraformaldeyde in $0.1 \mathrm{M}$ phosphate buffer (PBS) at $4^{\circ} \mathrm{C}$ for several days. Biocytin was revealed with avidin biotin complex (Elite $\mathrm{ABC}$ kit, Vector Laboratories). Although axonal labelling between the GP and STN was observed in both planes of section, the most robust connectivity including labelling of axonal tracts and cell bodies was observed in $20^{\circ}$ parasagittal sections (Figure 1A). This plane of section was used for all subsequent electrophysiological recordings.

\subsection{Evoked Synaptic Currents}

Whole-cell recordings coupled with electrical stimulation were used to show connectivity between the GP and the STN. Whole-cell recordings were made using borosilicate glass pipettes of 3-6M $\Omega$ resistance containing (in $\mathrm{mM}$ ) K-gluconate $125, \mathrm{NaCl}$ $10, \mathrm{CaCl}_{2} 1, \mathrm{MgCl}_{2}$ 2, EGTA 0.5, HEPES 10, GTP 0.3, Mg-ATP 2, biocytin 5, adjusted to $\mathrm{pH} 7.25$ with $\mathrm{KOH}$. Individual neurons were visualized (x40 water immersion objective) using differential interference contrast infa-red microscopy (Olympus BX 501, Japan) with CCD camera (Hitachi KP-M1, Japan) and contrast enhancement system (ADV-2, Brian Reece Scientific Ltd, Newbury, UK). Membrane currents and potentials were monitored using an Axopatch 200B patch-clamp amplifier (Axon Instruments, Foster City, CA, USA). Synaptic events were evoked by bipolar single shock stimulation $(0.2 \mathrm{~ms}, 1-3 \mathrm{~mA})$ using a constant current stimulation unit (Digitimer, DS2A). 


\section{The GP-STN network in vitro}

Most GP cells recorded (>90\% of the population) corresponded to Type A neurons of the rat (Cooper \& Stanford, 2000), the type II cells of guinea pig (Nambu \& Llinás, 1994) and bursting cells of Nakanishi et al., 1987. They were easily identified by the presence of a time- and voltage-dependent 'sag' of membrane potential evoked by hyperpolarising current steps indicative of $\mathrm{I}_{\mathrm{h}}$, and anodal break rebound depolarisations, often accompanied by action potential firing (Cooper \& Stanford, 2000). All STN cells displayed both $\mathrm{I}_{\mathrm{h}}$ and rebound depolarisations, typical characteristics of STN neurons (Beurrier et al 1999, Bevan and Wilson 1999, Bevan et al 2000).

As there is no anatomical evidence for a GABA projection to the STN, other than from the GP, or a glutamate projection to the GP, other than the STN, which courses through the STN, we used single shock electrical stimulation $(0.2 \mathrm{~ms}, 1-3 \mathrm{~mA})$ within the STN or within the GP to evoke excitatory and inhibitory postsynaptic potentials.

Stimulation of the STN evoked bicuculline resistant EPSCs in the GP in 22/33 cells (66\%) (Figure 1Bi), which were blocked by the glutamate antagonists CNQX $(10 \mu \mathrm{M})$ and AP5 $(100 \mu \mathrm{M})(\mathrm{n}=5)$. Often responses with double peaks could be observed which could be due to the activation of polysynaptic circuitry, asynchronous release following single stimuli or the promotion of a somatic spike that may evoke further release.

Stimulation of the GP in the presence of CNQX $(10 \mu \mathrm{M})$ and AP5 $(100 \mu \mathrm{M})$ evoked $\mathrm{GABA}_{\mathrm{A}}$ receptor mediated IPSCs in the STN in 44/59 slices (75\%) which reversed close to theoretical equilibrium potential for chloride (Figure 1Bii). These postsynaptic currents were blocked by the $\mathrm{GABA}_{\mathrm{A}}$ antagonist bicuculline $(10 \mu \mathrm{M}, \mathrm{n}=7)$. In 2 of 2 slices evoked IPSCs, and evoked EPSCs were recorded, indicative of reciprocal connectivity.

\subsection{Functional connectivity}

STN-evoked EPSPs were able to trigger action potentials in the GP while single shock simulation in the GP evoked a single IPSP in the STN. Increasing the number of shocks induced IPSP summation and inhibition of STN action potential firing. The following rebound depolarisations were then able to elicit further action potential firing $(\mathrm{n}=4$, Figure 1C). These rebound depolarisations were not found to produce action potentials at any consistent frequency but may provide recurrent excitation of the GP in a reciprocally connected network required for reverberating oscillatory activity as previously proposed (Plenz and Kitai, 1999, Bevan et al 2000). However, regenerative rebound depolarisations were never observed, even when using stimulus trains.

\subsection{Spontaneous Synaptic Currents}

Evidence for spontaneous release of GABA and glutamate on both GP and STN cells was also observed. At a holding potential of $-50 \mathrm{mV}$, spontaneous outward currents indicative of sIPSCs (from the GP) were observed in 85/150 (57\%) STN cells. Inward currents indicative of sEPSCs (from the cortex, thalamus, pedunculopontine nucleus) were observed in 106/150 (71\%) cells. Inward and outward currents were often observed in the same recording (Figure 1D). In GP cells, sEPSCs (from the STN) were observed in 26/43 cells $(60 \%)$ while sIPSCs (from GP axon collaterals) were observed in all 43 cells recorded (Figure 1D). 
The GP-STN network in vitro

A i

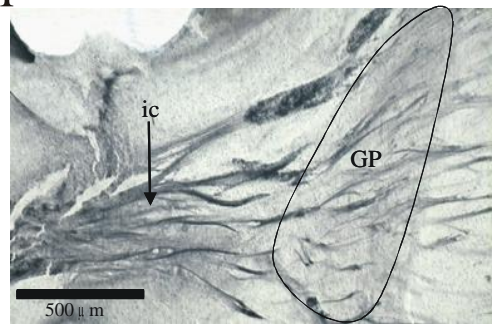

B

D GP
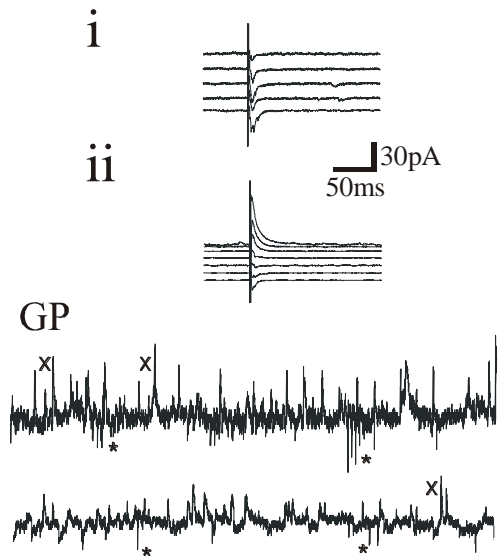

ii

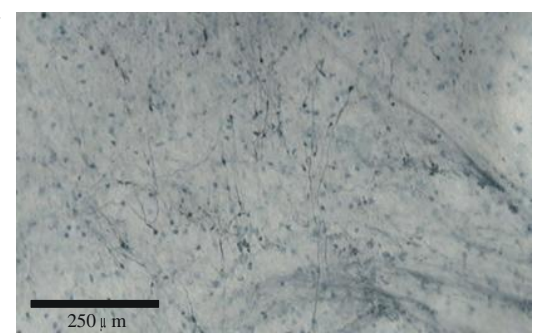

C

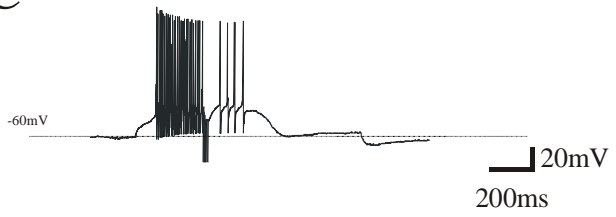

STN

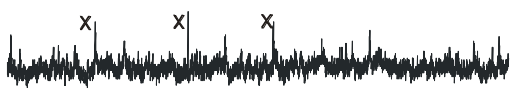

$-50 \mathrm{mV}$

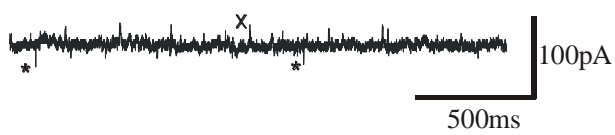

Figure 1

Ai. A parasagittal slice in which the STN was injected with biocytin showing labelled fibre tracts heading towards the GP and striatum. Aii Biocytin labelled cell bodies of the GP from the slice shown in Ai Bi. Current responses to single shock electrical stimulation in the STN and Bii in the GP at holding potentials -90 to $-30 \mathrm{mV}$ C. Voltage records from a STN cell held at $-60 \mathrm{mV}$. 300ms step depolarisations promoted fast action potential firing. 5 shocks at $200 \mathrm{~Hz}$ in the GP evoked IPSPs, which summated and blocked action potential firing. Following this inhibition there was a rebound depolarisation, which promoted further action potential firing. D. Spontaneous inward * and outward currents ${ }^{\mathrm{X}}$ recorded from a GP cell and an STN cell at holding potentials -40 and $-50 \mathrm{mV}$. 
The GP-STN network in vitro

\section{CONTROL SPIKE FIRING PATTERNS IN THE STN ARE INDEPENDENT OF GABA OR GLUTAMATE RELEASE}

Does the connectivity and GABA release from the GP play a role in the rate and pattern of spontaneous STN activity in the mouse slice preparation? To address this question we used extracellular single-unit recordings of STN activity using borosilicate glass pipettes of resistance $6-10 \mathrm{M} \Omega$ filled with $2 \mathrm{M} \mathrm{NaCl}$ and bath application of the $\mathrm{GABA}_{\mathrm{A}}$ antagonist picrotoxin $(50 \mu \mathrm{M})$ in order to eliminate any effects of synaptically released GABA. Single units were detected and amplified x10,000 with Axon Cyberamp 380 and AL402 differential amplifiers. Data acquisition and analysis was performed with a Micro-1401 mkII and Spike2 software (Cambridge Electronic Design). Single unit waveforms were discriminated from noise and sorted off-line.

In control slices, STN cells fire action potentials at a tonic rate of $9.24 \pm 0.92 \mathrm{~Hz}$. $(\mathrm{n}=82)$. Application of picrotoxin resulted in no effect on the frequency of all 9 STN cells tested indicating that at least under control conditions the tonic release of GABA has no effect on firing rate. To block any glutamatergic tone the glutamate antagonist CNQX $(10 \mu \mathrm{M})$ was applied. No change of firing rate was observed in all 8 STN cells indicating that at least under control conditions the tonic release of glutamate has no effect on firing rate.

\section{NMDA AND APAMIN-INDUCED BURST FIRING IN THE STN IS ALSO INDEPENDENT OF GLUTAMATE AND GABA RELEASE}

In an attempt to replicate the bursting activity observed in STN neurons during dopamine depletion in vivo and to increase the release of GABA from the GP, we bath applied the ionotropic glutamate receptor agonist NMDA (Zhu et al., 2004). NMDA $(20 \mu \mathrm{M})$ induced an increase in firing rate of $415 \pm 103 \%(\mathrm{n}=15)$. However, only $3 / 18$ cells $(17 \%)$ changed the firing pattern from regular to a burst-firing. Bursting activity was much more reproducible if NMDA $(20 \mu \mathrm{M})$ was applied in conjunction with the calcium-activated potassium channel blocker, apamin $(20-100 \mathrm{nM})$. Apamin has previously been shown to enhance NMDA-mediated burst firing, both in dopaminergic neurons (Seutin et al., 1993)

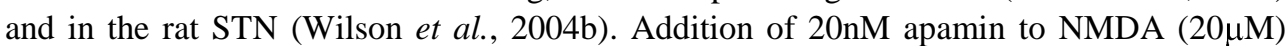
induced bursting in 5/19 STN cells (26\%), while 50nM apamin induced bursting in 12/25 cells (48\%), and 100nM apamin in 24/44 cells (55\%).

The burst parameters of 25 STN cells were analysed using the program of Kaneoke Y \& Vitek J.l. (1996). This burst detection algorithm identifies cells which fire in patterns which differed significantly from the Poisson distribution with a mean of 2 , thus giving a burst index $>0.5$. No differences in the burst firing induced by different concentrations of apamin were observed. Thus, analysis of pooled data revealed slow oscillatory bursting at a frequency of $0.46 \pm 0.06 \mathrm{~Hz}$, each burst containing $31.7 \pm 5.35$ spikes, the interspike interval within bursts being $24.2 \pm 3.73 \mathrm{~ms}(\mathrm{n}=25)$. Such bursting activity would be expected to promote increased GABA or glutamate release in the STN and GP respectively. Indeed, 6/12 single unit recordings from GP cells show NMDA/apamin induced burst firing. 


\section{The GP-STN network in vitro}

As burst firing induces the release of more transmitter than single spiking (Lisman, 1997) and STN cells display a reverse spike frequency adaptation and a steep secondary range in their frequency-intensity curves (Wilson et al., 2004a) and are therefore more sensitive to synaptic input when excited in the burst range, we expected to observe more pronounced effects of applied picrotoxin and CNQX. However, this was not the case. Using the whole cell technique, picrotoxin $(50 \mu \mathrm{M})$ was applied to $5 \mathrm{NMDA} / \mathrm{apamin}$ induced bursting cells, which all continued to burst fire with no significant change in oscillation frequency $(\mathrm{P}=0.86)$, spikes per burst $(\mathrm{P}=0.86)$, or ISI within bursts $(\mathrm{P}=0.5)$. CNQX $(100 \mu \mathrm{M})$ was added to 6 bursting cells. All cells continued to burst fire with no significant change in oscillation frequency $(\mathrm{P}=0.32)$, spikes per burst $(\mathrm{P}=0.44)$, or ISI within bursts $(\mathrm{P}=0.22)$. Thus, despite $75 \%$ of our GP cells being connected to the STN and $66 \%$ of STN cells being connected to the GP and extensive evidence for sIPSCs and sEPSCs in our mouse slice preparation, we have no evidence that GABA or glutamate has any role in promoting, shaping or modulating the NMDA/apamin induced burst-firing patterns in STN neurons. Rather, the bursting observed is likely to be purely intrinsically driven within the somatodendritic segments of the individual cells studied.

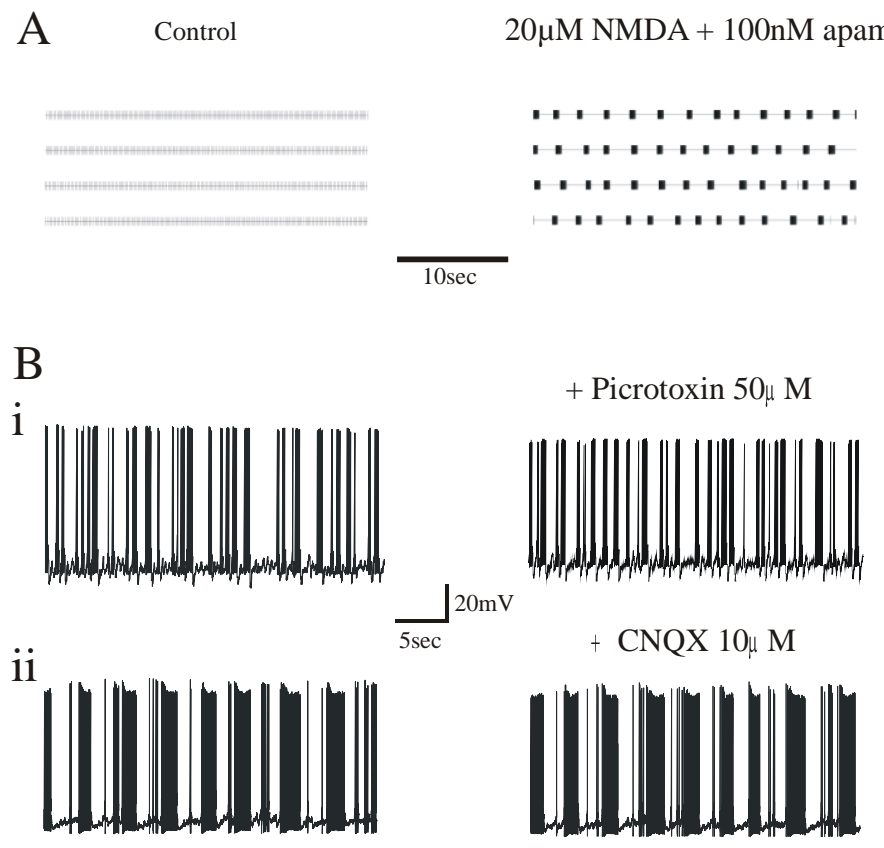

Figure 2

A. Extracellular single-unit recording from a spontaneously firing STN neuron in control and following perfusion with NMDA $(20 \mu \mathrm{M})$ and apamin $(100 \mathrm{nM})$. B. Whole cell recording from two STN cells bursting in the presence of NMDA and apamin. Bath application of Bi picrotoxin $(50 \mu \mathrm{M})$ or Bii CNQX (10 $\mu \mathrm{M})$ had no effect on the oscillation frequency, number of spikes per burst or the interspike interval (ISI) within bursts. 


\section{The GP-STN network in vitro}

\subsection{Paired Recordings}

Simultaneous single unit recordings of neuronal activity were made from a number of cells in the STN and the GP. 7 pairs of regularly firing STN/GP neurons were recorded. In the presence of NMDA $(20 \mu \mathrm{M})$ and apamin $(100 \mathrm{nM})$, bursting of both cells was observed in 3 of the 7 pairs (Figure 3A). In 3 pairs, only the STN exhibited bursting activity and in one pair bursting was promoted only in the GP unit. In all cases the activity observed remained totally uncorrelated. 7 pairs of bursting STN neurons were also recorded. In each pair, the regular/tonic activity in control conditions was uncorrelated as was the bursting activity induced by NMDA $(20 \mu \mathrm{M})$ and apamin $(100 \mathrm{nM})$ application (Figure 3B).

A.

STN/STN

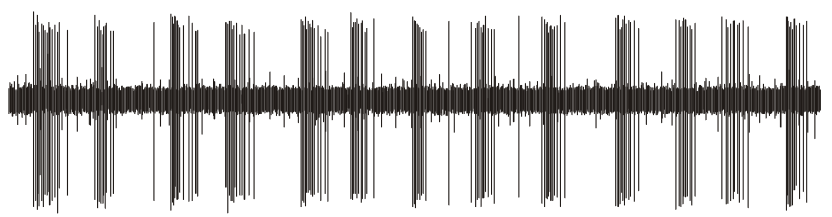

GP

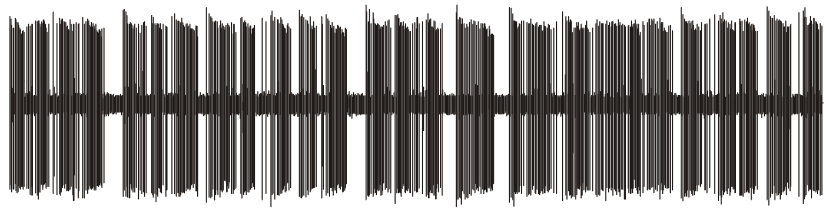

B.
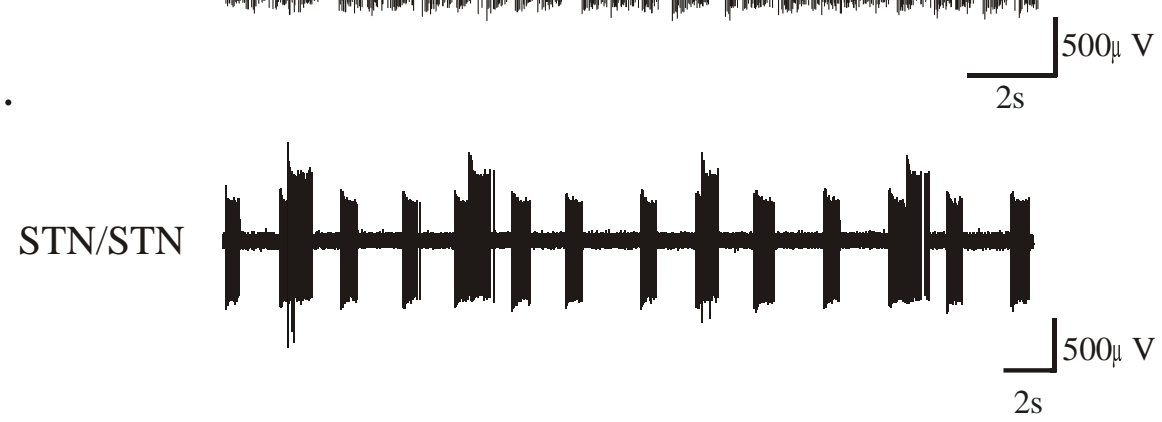

Figure 3

Simultaneous single- unit recordings from STN and GP neurons in the presence of NMDA and apamin showing uncorrelated bursting activity. A. A bursting STN cell and a bursting GP cell. Note the presence of a second nonbursting unit within the STN recording. B. Two bursting STN units recorded on a single electrode. 


\section{The GP-STN network in vitro}

\section{STUDIES IN DOPAMINE DELETED ANIMALS}

As adaptive changes caused by chronic dopamine depletion may be a fundamental requirement for the manifestation of synchronous oscillatory activity we repeated the extracellular studies in slices obtained from MPTP treated mice. 8 mice were treated 10-21 days previously with the dopamine neurotoxin 1-methyl-4-phenyl-1,2,3,6tetrahydropyridine (MPTP), using the regime described by Araki et al. (2001). Single-unit extracellular recordings were made from up to 3 spontaneously firing STN neurons simultaneously. STN neurons in slices from MPTP-lesioned animals fire at $4.23 \pm 0.35 \mathrm{~Hz}$ $(n=78)$, significantly slower than those from control animals $(9.78 \pm 1.16, n=20$; $\mathrm{p}<0.0001)$. STN neurons from MPTP lesioned animals fire irregularly, with an average coefficient of variation of inter-spike interval of $94.4 \%(n=78)$, significantly higher $(\mathrm{p}=0.0007)$ than that seen in neurons from control animals $(25.8 \%, \mathrm{n}=33)$. However, firing in simultaneously recorded STN-STN (20 pairs), STN-GP (8 pairs) and GP-GP neurons (3 pairs) were all uncorrelated. Furthermore, as in control slices, there was no significant change in rate or pattern of firing on application of CNQX $(10 \mu \mathrm{M})$, AP5 $(100 \mu \mathrm{M})$ and picrotoxin $(50 \mu \mathrm{M})$.

\section{CONCLUSIONS}

An in vitro mouse slice preparation from control and MPTP treated mice in which functional GP-STN connectivity is maintained does not produce oscillatory bursting activity in GP-STN networks without pharmacological interventions and when induced to burst does not require GABA or glutamate release to maintain the activity. Thus, we have no evidence that the functionally connected but isolated GP - STN network can act as a pacemaker for synchronous correlated activity in the basal ganglia and must conclude that other inputs such as those from cortex and/or striatum are required.

\section{ACKNOWLEDGEMENTS}

This study was supported by The Wellcome Trust project grants 068818 (IMS) and Research Leave Fellowship 063461 (MGL), The Parkinson's Disease Society and a research grant from the Royal Society. IMS is a MRC career establishment fellow.

\section{REFERENCES}

Albin, R.L., Young, A.B., Penney, J.B. (1989) The functional anatomy of basal ganglia disorders. Trends Neurosci., 12, 366-375.

Araki T, Mikami T, Tanji H, Matsubara M, Imai Y, Mizugaki M, Itoyama Y. (2001) Biochemical and immunohistological changes in the brain of 1-methyl-4-phenyl-1,2,3,6-tetrahydropyridine (MPTP)treated mouse. Eur J Pharm Sci 12, 231-8.

Bergman H, Wichmann T, Karmon B, Delong MR (1994) The primate subthalamic nucleus. II. Neuronal activity in the MPTP model of parkinsonism. J Neurophysiol 72, 507-520.

Beurrier C, Congar P, Bioulac B, Hammond C (1999). Subthalamic nucleus neurons switch from single-spike activity to burst-firing mode. J Neurosci 19, 599-609.

Bevan MD, Wilson CJ (1999) Mechanisms underlying spontaneous oscillation and rhythmic firing in rat subthalamic neurons. J Neurosci 19, 7617-7628. 


\section{The GP-STN network in vitro}

Bevan, M.D., Wilson, C.J., Bolam, J.P., Magill, P.J. (2000) Equilibrium potential of $\mathrm{GABA}_{\mathrm{A}}$ current and implications for rebound burst firing in rat subthalamic neurons in vitro. J Neurophysiol., 83, 31693172.

Bevan, M.D., Magill, P.J., Hallworth, N.E., Bolam, J.P. and Wilson, C.J. (2002) regulation of the timing and pattern of action potential generation in rat subthalamic neurons in vitro by GABA-A IPSPs. J. Neurophysiol., 87, 1348-1362.

Cooper, A.J., Stanford, I.M. (2000) Electrophysiological and morphological characteristics of three subtypes of rat globus pallidus neuron in vitro. J Physiol., 527.2, 291-304.

DeLong, M. (1990) Primate models of movement disorders of basal ganglia origin. Trends Neurosci., 13 (7), 281285.

Filion, M., Tremblay, L. (1991) Abnormal spontaneous activity of globus pallidus neurons in monkeys with MPTP-induced parkinsonism. Brain Res., 547, 142-151.

Johnson, S.W, Seutin, V. (1997) Bicuculline methiodide potentiates NMDA-dependent burst firing in rat dopamine neurons by blocking apamin-sensitive $\mathrm{Ca}^{2+}$ - activated $\mathrm{K}^{+}$currents. Neurosci Letts., 231, 1316

Kaneoke, Y., Vitek, J.L. (1996) Burst and oscillation as disparate neuronal properties. J Neurosci Methods, 68, 211-223.

Lisman JE (1997). Bursts as a unit of neural information: Making unreliable synapses reliable. Trends Neurosci 20, 38-43.

Magill, P.J., Bolam, J.P., Bevan, M.D. (2000) Relationship of activity in the subthalamic nucleus-globus pallidus network to cortical electroencephalogram. J Neurosci., 20, 820-833.

Magill, P.J., Bolam, J.P., Bevan, M.D. (2001) Dopamine regulates the impact of the cerebral cortex on the subthalamic nucleus-globus pallidus network. Neurosci., 106, 313-330.

Magnin, M., Morel, A., Jeanmonod, D. (2002) Single unit analysis of the pallidum, thalamus, and subthalamic nucleus in Parkinsonian patients. Neurosci. 96, 549-564.

Nakanishi, H., Kita, H., Kitai, S.T. (1987) Electrical membrane properties of rat subthalamic neurons in an in vitro slice preparation. Brain Res., 437, 35-44.

Nambu, A., Llinás, R. (1994) Electrophysiology of globus pallidus neurons in vitro. J Neurophysiol., 72, 11271139.

Nini, A., Feingold, A., Slovin, H. \& Bergman, H. (1995) Neurons in the globus pallidus do not show correlated activity in the normal monkey, but phase-locked oscillations appear in the MPTP model of parkinsonism. J. Neurophysiol., 74, 1800.

Plenz, D., Kitai, S.T. (1999). A basal ganglia pacemaker formed by the subthalamic nucleus and external globus pallidus. Nature, 400, 677-682.

Seutin, V., Johnson, S.W., North, R.A. (1993). Apamin increases NMDA-induced burst-firing of rat mesencephalic dopamine neurons. Brain Res., 630, 341-344.

Wilson CJ, Weyrick A, Terman D, Hallworth NE, Bevan MD (2004a). A model of reverse spike frequency adaptation and repetitive firing of subthalamic nucleus neurons. J.Neurophysiol 91,1963-1980.

Wilson CL, Puntis M, Lacey MG (2004b). Overwhelmingly asynchronous firing of rat subthalamic nucleus neurons in brain slices provides little evidence for intrinsic connectivity. Neuroscience 123, 187-200.

Raz, A., Vaadia, E. \& Bergman, H. (2000) Firing Patterns and Correlations of Spontaneous Discharge of Pallidal Neurons in the Normal and the Tremulous 1-Methyl-4-Phenyl-1,2,3,6-Tetrahydropyridine Vervet Model of Parkinsonism. J. Neurosci., 20, 8559-8571.

Raz, A., Frechter-Mazar, V., Feingold, A., Abeles, M., Vaadia, E. \& Bergman, H. (2001) Activity of pallidal and striatal tonically active neurons is correlated in MPTP-treated monkeys but not in normal monkeys. J. Neurosci., 21, RC128 (1-5).

Zhu, Z.T., Munhall, A., Shen, K.Z., Johnson, S.W. (2004). Calcium-dependent subthreshold oscillations determine bursting activity induced by N-methyl-D-aspartate in rat subthalamic neurons in vitro. Eur. J. Neurosci., 19, 1296-1304. 